\title{
Medievalista
}

Online

13 | 2013

Número 13

\section{De l'image à l'imaginaire médiéval}

\section{Philippe Walter}

\section{OpenEdition}

\section{Journals}

Édition électronique

URL : http://journals.openedition.org/medievalista/539

DOI : 10.4000/medievalista.539

ISSN : 1646-740X

\section{Éditeur}

Instituto de Estudos Medievais - FCSH-UNL

\section{Référence électronique}

Philippe Walter, «De l'image à l'imaginaire médiéval », Medievalista [En ligne], 13| 2013, mis en ligne le 19 février 2014, consulté le 30 avril 2019. URL : http://journals.openedition.org/medievalista/539

DOI : 10.4000/medievalista.539

(C) IEM 
Título: De l'image à l'imaginaire médiéval

Autor(es): Philippe Walter

Enquadramento Institucional: Université de Grenoble, Grenoble, France

Contacto: philippe.walter@wanadoo.fr

Fonte: Medievalista [Em linha]. №13, (Janeiro - Junho 2013). Dir. José Mattoso. Lisboa:

IEM.

Disponível em: http://www2.fcsh.unl.pt/iem/medievalista/

ISSN: $1646-740 \mathrm{X}$

\section{Resumo}

Diante do poder esmagador das imagens mediáticas nas sociedades contemporâneas seremos capazes de conceber o lugar da imagem na Idade Média? Como perspectivar o seu valor fora da lógica de sobrevalorização radical do domínio do visual a que estamos diariamente sujeitos? Como compreender a complexidade da sua função sem incorrer de imediato em anacronia? Reconstituindo uma breve história da Imagem, desde os alvores do platonismo, na Grécia Antiga, à consolidação do cristianismo, dar-se-á conta da progressiva emergência do imaginário medieval, afirmação da razão sensível na base de um pensamento que não apenas dispõe de conceitos mas mobiliza afectos. Indagar-se-á como ocorre o processo de simbolização da imagem e como, por meio dele, se cruzam e articulam o visível e o invisível, a percepção e a interpretação, a memória e a promessa do porvir, o passado e o futuro.

Palavras-chave: Imagem, iconoclasmo, Idade Média, imaginário medieval, visual 


\section{Abstract}

Facing the mediatic image's crushing power in contemporary societies, can we conceive the locus of image in Middle Ages? How are we able to enframe it's value out of a radical overvaluation of the visual that daily submits us? How to understand it's functions complexity without immediate anacronism? While reconstituting a brief Image's history, from the dawn of platonism, in Ancient Greece, up to the consolidation of Christianity, this text shall address the formation of a medieval imaginary, grounding on the affirmation of sensible reason a thought that not only uses concepts but also mobilizes affects. It will question how the process of image's symbolization occurs and how, through it, concepts like invisible and visible, perception and interpretation, memory and the promise of what's to come, pass and future, are connected and articulate.

Keywords: Image, iconoclasm, Middle Ages, medieval imaginary, visual

\section{De l’image à l’imaginaire médiéval'1}

\section{Philippe Walter}

Le monde contemporain voit le triomphe absolu de l'image sous toutes ses formes. Aujourd'hui, cinéma, télévision et internet ont banalisé les images d'autant plus facilement que les moyens techniques de les fabriquer et de les reproduire à l'infini sont

\footnotetext{
${ }^{1}$ Conferência proferida no âmbito do Seminário de Doutoramento "Imagem Medieval, investigação e reflexão interdisciplinar", organizado pelo Instituto de Estudos Medievais FCSH - UNL, no primeiro semestre do ano lectivo de 2010, 2011.
} 
désormais à la portée de chacun. Par ailleurs, sur le plan de la connaissance, l'empire du visible repousse sans cesse de nouvelles limites. Aujourd'hui, l'homme peut voir l'infiniment petit (grâce au microscope) et l'infiniment loin (grâce au téléscope). Il peut faire reculer les frontières du visible en voyant le cerveau fonctionner. Le sociologue Edgar Morin prétend que le cinéma et le règne de l'image ont transformé l'homme organiquement et intellectuellement: «Alors que le seizième siècle avant de voir «entend et flaire, hume les souffles et capte les bruits» comme l'a monté L. Febvre dans son Rabelais, le cinéma nous révèle la décadence de l'ouïe (inadéquation de la source sonore aux sources visuelles, approximations du doublage, schématisation du mixage, etc...) en même temps qu'il assied son empire à partir des pouvoirs concrets et analytiques de l'œil»².

Grâce aux techniques de plus en plus sophistiquées de l'image, aujourd'hui nous sommes partout et nous voyons tout. Notre œil est devenu notre repère essentiel dans l'univers, bien plus que l'ouïe. Alors que les sociétés traditionnelles du Moyen Age, vivaient sous le règne de la parole et de l'ouïe (qui entretient la tradition orale des contes, rumeurs et légendes) ${ }^{3}$, le monde moderne et post-moderne vit sous l'emprise permanente et aveuglante de l'image ${ }^{4}$. Cette dernière a largement contribué à tuer la tradition orale et contribué en atrophiant notre culture auditive (qui sait encore reconnaître aujourd'hui les différents chants d'oiseaux alors que les hommes du Moyen Age ne les confondaient nullement?). Ceci n'est pas sans conséquences sur l'imaginaire littéraire: les derniers conteurs traditionnels ont disparu dans les Alpes au moment où la télévision a envahi les foyers. Aujourd'hui, il n'est plus possible de recueillir des contes issus de la tradition orale car les conteurs les ont oubliés. La télévision a affaibli les pouvoirs de l'ouïe et la mémorisation qui s'y attachait. En faisant régner partout l'image et la culture visuelle, elle a aussi changé la valeur des mots et des choses.

Après ce constat, il apparaît que le premier danger, lorsqu'on étudie l'image médiévale, peut être celui de l'anachronisme. Il consiste à regarder l'image médiévale avec nos yeux modernes et à donner à l'image les mêmes significations et fonctions

\footnotetext{
${ }^{2}$ Morin, E. - Le cinéma ou l'homme imaginaire. Essai d'anthropologie, Paris: Gonthier, 1958, p. 176.

${ }^{3}$ Voir sur ce point les travaux de Zumthor, P. - Introduction à la poésie orale, Paris, Seuil, 1986 et La lettre et la voix, Paris: Seuil, 1987.

${ }^{4}$ Wunenburger, J. J. - L'homme à l'âge de la télévision, Paris: PUF, 2001.
} 
qu'aujourd'hui. Or, la question de l'image (et de la culture visuelle) au Moyen Age exige, pour être correctement traitée, des investigations pluridisciplinaires. Encore fautil s'aviser qu'un historien ${ }^{5}$, un anthropologue ${ }^{6}$, un historien de $1^{\prime} \operatorname{art}^{7}$, un philosophe ${ }^{8}$, un critique littéraire ${ }^{9}$ 'accordent pas le même sens au mot image. Il faut commencer par définir la notion pour espérer tirer profit d'une réflexion à la croisée de plusieurs disciplines. De quoi parle-t-on quand on emploie le mot ymage au Moyen Age ? Comment l'image est-elle devenue à cette époque le fondement d'un imaginaire qui a fortement marqué la littérature?

\section{Un préalable philologique}

Que signifie le mot «image» au Moyen Age? Les dictionnaires de la langue médiévale sont très clairs sur le sujet. ${ }^{10}$ Une image est une "statuette". Rien de plus, rien de moins. Il en est de même en portugais médiéval et jusqu'en espagnol classique où imagem désigne encore un tableau et une statue (Thérèse d'Avila passe devant une «image» du Christ crucifié et se convertit). L'étymologie du mot image est latine. Imago désigne l'image et ensuite "une représentation, un portrait, un fantôme (dans la langue poétique), une apparence (par opposition à la réalité)"11. Plus précisément, l'imago est à Rome un portrait d'ancêtre, une image de cire que l'on plaçait dans l'atrium et que l'on portait

\footnotetext{
5 Baschet, J. et Schmitt, J.C. éd. - L'image : fonction et usages des images dans l'Occident médiéval, Paris: Le Léopard d'or, 1996.

${ }^{6}$ Belting, H. - Image et culte: une histoire de l'image avant l'époque de l'art, Paris: Cerf, 1998 (traduction de : Bild und Kunst : eine Geschichte des Bildes vor dem Zeitalter der Kunst).

${ }^{7}$ Garnier, F. - Le langage de l'image au Moyen Age, I. Signification et symbolique, Paris: Le Léopard d'or, 1989. II. Grammaire des gestes, Paris: Le Léopard d'or, 1989. Wirth, J. - L'image médiévale: naissance et développements, Paris: Klicksieck, 1989. L’image à l'époque romane, Paris: Cerf, 1999. L'image à l'époque gothique (1140-1280), Paris: Cerf, 2008.

${ }^{8}$ Lemoine, M. éd., - L'image dans l'art et la pensée du Moyen Age, Turnhout: Brepols, 2006.

Boulnois, O. - Au-delà de l'image. Une archéologie du visuel au Moyen Age (Ve-XVIe siècle), Paris: Seuil, 2008.

${ }^{9}$ Caminade, P. - Image et métaphore: un problème de poétique contemporaine, Paris: Bordas, 1970.

${ }^{10}$ W. von Wartburg - Französisches Etymologisches Wörterbuch, Bâle: Helbing et Lichtenbahn, 1952, t. 4, p. 564-566, s.v. Imago. En ancien français « statue, figure moulée ou sculptée » (dans la Vie d'Alexis et la Chanson de Roland) mais aussi «portrait peint ou dessiné de quelqu'un ou quelque chose » (Chrétien de Troyes, Cligès). Le sens de «réflexion d'un objet sur un miroir » est attesté chez Marie de France (dans ses Fables).

${ }^{11}$ Ernout, A. et Meillet, A. - Dictionnaire étymologique de la langue latine, Paris: Klincksieck, 1967, p. 309.
} 
aux funérailles. ${ }^{12}$ En rhétorique, l'image désigne le procédé de style qui utilise une comparaison. Le mot correspond au grec eikon et à fantasma (on parle aussi de figura, c'est-à-dire de «figure» de rhétorique, ce qui tendrait à prouver que l'image est à la base de toute le rhétorique). Imago est en lien avec le verbe imitor, "chercher à reproduire l'image, imiter". Le sens latin d'imago semble a priori plus large (il désigne toute image quelle qu'elle soit) alors que le sens médiéval de statuette est plus restreint. Mais, dans un cas comme dans l'autre, il est clair que l'on se trouve toujours devant une reproduction, une fabrication, une imitation ou une représentation de la réalité. L'image n'est pas l'être ou l'essence des choses, elle n'en est que le substitut. Ceci est un point fondamental dans la théorie occidentale de la connaissance, telle qu'elle s'élabore avec la philosophie grecque.

\section{L'iconoclastie occidentale}

Très tôt était apparue pour les Grecs de l'antiquité une «faiblesse» congénitale de l'image dans la quête de la connaissance. ${ }^{13}$ Un mythe célèbre illustre cette vérité. Narcisse est victime des pouvoirs dangereux de l'image (à travers le thème du miroir). Pour avoir vu son ombre reflété à la surface de l'eau, Narcisse tombe amoureux de luimême. Il est amoureux de son propre reflet qu'il prend pour l'image d'un autre. Mais cette image que lui renvoie l'eau le piège et le fait sombrer dans la mort. Pour lui, l'image est une illusion fatale. De manière générale, elle est un mirage trompeur qui ne nous apprend rien sur nous-même ni sur le monde. En grec narkè signifie «engourdissement, torpeur causée par la paralysie, le froid, l'effroi» ${ }^{14}$ et le lien avec le nom de Narcisse a été établi par Plutarque ${ }^{15}$, accréditant ainsi la valeur tragique de l'image mortifère.

Une fable philosophique célèbre aboutit au même constat. Le mythe platonicien de la caverne présentait les hommes comme d'éternelles victimes du mirage incessant des

\footnotetext{
${ }^{12}$ Gaffiot, F. - Dictionnaire illustré latin français, Paris: Hachette, 1934, p. 773.

${ }^{13}$ Vernant, J. P. - Religions, histoires, raisons, Paris: 10/18, 1979, p. 105-137.

${ }^{14}$ Chantraine, P. - Dictionnaire étymologique de la langue grecque. Histoire des mots, Paris: Klincksieck, 1968 , p. 735.

${ }^{15}$ Chantraine, P. - Dictionnaire étymologique de la langue grecque, p. 736.
} 
images. La condition humaine est prisonnière des simulacres et des apparences alors que les Idées essentielles lui restent inaccessibles. Les Grecs inventèrent alors cette discipline très particulière de l'esprit qui s'appelle "philosophie". Il s'agissait d'un exercice mental pour s'entraîner à résister aux illusions du monde et aux images fausses qu'il nous prodigue si généreusement. Il s'agissait, grâce à l'ascèse philosophique, de tendre à la perfection de l'activité mentale fondée sur l'exercice de l'activité critique, seule garantie d'une vraie connaissance. La recherche des idées vraies est antinomique de la contemplation des images. L'image n'est qu'une semblance, un faux-semblant. L'image ne relève pas de l'être mais du paraître. Elle «accomplit son œuvre loin de la vérité» selon une expression platonicienne de la République.

Ce qu'établit la philosophie grecque aux origines de la culture occidentale, les religions monothéistes vont le reconnaître à leur tour et, tout particulièrement le judaïsme qui va influencer sur ce point l'Islam et le christianisme (au moins à ses débuts). L'Ancien Testament interdit toute image de Dieu dans le Décalogue: «Tu n'auras pas d'autre Dieu que moi. Tu ne te feras aucune image sculptée, rien qui ressemble à ce qui est dans les cieux là-haut, ou sur la terre ici-bas, ou dans les eaux au-dessous de la terre. Tu ne te prosterneras pas devant devant ces images ni ne les serviras, car moi, Yahvé, ton Dieu, je suis un Dieu jaloux» (Exode, 20, 2-5). Le judaïsme ne tolère aucune représentation du divin. Il refuse même de prononcer le nom de Yahvé en le transcrivant sous la forme du tétragramme imprononçable. Le christianisme de saint Augustin reconnaît encore que l'image parfaite (celle de Dieu) est invisible. Dieu ne peut être saisi ni figuré par aucune image ni par aucun signe en général (par exemple la parole). ${ }^{16}$ «La vérité se voit, non

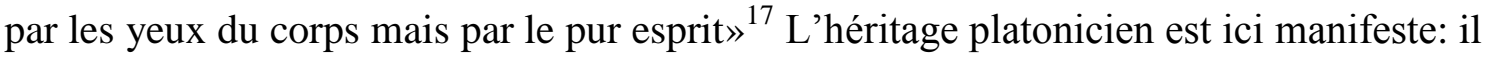
rejoint celui de la Bible. On peut donc parler avec Gilbert Durand d'une véritable iconoclastie occidentale. ${ }^{18}$

\footnotetext{
${ }^{16}$ Kessler, H. L. - Spiritual seeing. Picturing God's invisibility in medieval art, Philadelphie: University of Pennsylvania Press, 2000.

17 Augustin - La Vraie religion, III, 3 (cité dans O. Boulnois, p. 58).

18 Durand, G. - «L'occident iconoclaste. Contribution à l'histoire du symbolisme », Cahiers internationaux de symbolisme, 2, 1963, p. 3-18.
} 


\section{L’image rédemptrice}

Le Moyen Age introduit une autre rupture importante par rapport à la culture antique. Si le platonisme dévalorisait les images, le christianisme va leur donner un pouvoir nouveau. C'est le christianisme oriental qui introduisit une révolution mentale riche de conséquences pour la culture occidentale. L'orthodoxie place l'image au cœur de la relation des hommes avec Dieu. L'icône y est vénérée car elle permet d'accéder au divin. Elle donne accès à l'inaccessible. Elle appelle l'être humain à s'ouvrir vers l'infini du divin. L'image retrouve alors un statut positif mais il est strictement limité à la sphère religieuse. Il en sera de même dans le christianisme occidental. Les images sont d'abord religieuses (illustration des Bibles manuscrites, fresques, sculptures, etc.).

Les chansons de geste (comme les récits hagiographiques) font allusion à la destruction des idoles (ymages) «sarrasines» (païennes). C'est reconnaitre implicitement le pouvoir de l'image dans le culte religieux (qu'il soit païen ou chrétien). En effet, pour le Moyen Age oriental puis occidental, l'image devient une présence médiatrice du divin. C'est par elle que transitent les messages d'imploration à Dieu, à la Vierge et à ses saints. C'est auprès d'elle qu'on vient se recueillir. Ici, l'attitude de Tristan peut apparaitre à certains égards comme religieuse ; il se recueille devant l'image d'Yseut comme devant l'image de la Vierge Marie par exemple. Tristan vit ainsi l'amour humain comme s'il s'agissait d'un amour divin.

$\mathrm{Au}$ XIIIe siècle triomphe le thème de l'image de la Vierge. Les Miracles de Notre Dame écrits par Gautier de Coinci (1177-1236) contiennent plusieurs épisodes d'images («statues») miraculeuses de la Vierge Marie. La Vierge y apparaît dans ces contes comme une médiatrice privilégiée entre l'homme et Dieu mais aussi entre l'homme et la société. Elle sait se montrer compatissante envers tous ceux qui viennent l'implorer humblement. Dans plusieurs de ces contes mariaux, c'est la statue (image) de Notre Dame qui joue le rôle principal. C'est à partir de la statue que se construit le récit. Dans un de ces miracles, un juif de Constantinople se moque d'une représentation de la Vierge Marie que viennent adorer les Chrétiens. Il jette la statue à terre et meurt aussitôt. Un chrétien ramasse la statue puis la nettoie. Une huile sainte se met alors à 
suinter de celle-ci et ce liquide miraculeux a de merveilleuses propriétés guérisseuses. ${ }^{19}$ Dans un autre miracle, une sainte femme a fondé un petit monastère où elle accueille un jour un moine de Constantinople en pèlerinage vers Jérusalem. Elle le charge de ramener de là-bas une statuette de la Vierge qui pourra trôner dans son monastère. Le moine s'exécute. Il achète cette statue qui va ensuite le protéger durant son voyage de retour. Elle va apaiser un lion féroce qui menaçait de le dévorer. Elle va aussi éloigner des brigands qui s'apprêtaient à le détrousser. Elle va enfin éloigner une tempête qui s'était abattue sur le navire qui le ramenait chez la pieuse femme. Ici encore, une liqueur suinte de la statue et provoque miracles et guérisons. ${ }^{20}$

Ces deux miracles ne font pas référence gratuitement à la ville de Constantinople. Il s'agit de la capitale du christianisme orthodoxe où s'était élevée une importante querelle théologique au $8^{\mathrm{e}}$ siècle. Elle opposait les iconoclastes et les iconodules. En 726, l'empereur byzantin Léon III avait ordonné la destruction de toutes les icônes de son empire. Saint Jean Damascène avait expliqué que cette destruction aboutissait à nier l'incarnation. Au siècle suivant, les iconodules imposent leur position. Depuis lors, le monde orthodoxe honore les icônes avec dévotion. Dans son esprit, l'image est la représentation de l'invisible à travers les choses visibles. Cette position tranche avec celle de l'Islam pour qui toute représentation du divin est interdite.

On sait tout ce que l'art chrétien d'Occident doit au christianisme oriental, bien avant l'union rompue avec Rome en 1054. L'influence du monde byzantin sur l'art religieux d'Occident est capitale. Cette influence se poursuit bien au-delà du Moyen Age puisque c'est la tradition iconodule née à Byzance qui serait aux origines de notre imaginaire contemporain selon M. J. Mondzain. ${ }^{21}$ Le courant iconoclaste qui parcourt toute la culture occidentale et qui fait dire à Pascal au XVIIe siècle que l'imagination (la faculté de créer des images) est maîtresse d'erreur et de fausseté n'a pu se trouver progressivement contesté que par un courant au moins aussi ancien qui faisait de l'image un instrument irremplaçable de connaissance. L'histoire de la plus sacrée des

${ }^{19}$ Gautier de Coinci - Miracles de Notre Dame, éd. de F. Koenig, Genève et Paris: Minard et Droz, 1970, t. 2, p. 101-104.

${ }^{20}$ Gautier de Coinci - Miracles de Notre Dame, éd. F. Koenig, Genève et Paris: Minard et Droz, 1970, p. $378-411$.

${ }^{21}$ Mondzain, M. J. - Image, icône, économie. Les sources byzantines de l'imaginaire contemporain, Paris: Seuil, 1996. 
images, celle du Christ en personne, mérite ici d'être rappelée car elle explique l'évolution capitale qu'apporte le christianisme médiéval par rapport à la conception antique de l'image.

Un portrait (ou ymage) de Jésus était conservé à Edesse (en actuelle Turquie, près de la frontière syrienne $)^{22}$. Selon un texte du IVe siècle (la Doctrine d'Addai), Abgar, roi d'Edesse, aurait fait parvenir à Jésus une lettre lui demandant de le guérir et lui offrant l'hospitalité. Mais comme l'heure était venue pour le Christ de remonter vers son Père, Hannan, l'envoyé du roi, fit du Sauveur un portrait de «couleurs choisies» pour en conserver le souvenir et il le rapporta à son roi. Pour Moïse de Khorène (470), Edesse possédait une image du Sauveur qui n'avait pas été faite de main d'homme. Pour Evagrius (593), l'image avait Dieu pour auteur. Pour Jean Damascène, docteur de l'Eglise grecque (au VIIIe siècle), c'est Hannan qui avait entrepris ce portrait ; l'éclat surnaturel et la mobilité des traits rendaient le visage divin insaisissable. Mais Jésus, s'emparant de la toile réfractaire au pinceau, l'appliqua sur son visage et y laissa son empreinte. Selon certaines variantes, il demanda de l'eau, se lava le visage et l'essuya, ce qui produisit le même résultat. A partir de 829, c'est la sueur du Christ qui est essuyée; dès 950 on précise qu'il s'agit de la sueur sanglante de Gethsémani. La tradition du «sidoine» est née. Entre les mains de Véronique, le linge portant l'image sanglante du Christ guérit l'empereur Vespasien de la lèpre. Il devient l'image parfaite, la vraie icone (vera icona), c'est-à-dire Véronique. A Rome, on conserve le voile de Véronique. Dante, l'évoque dans Le Paradis (XXXI, 103-105) de sa Divine Comédie:

Comme un homme, venu, qui sait? de Croatie/ Jusque chez nous, pour voir la Véronique, / Ne peut en assouvir sa faim invétérée :Mais en pensée dit, tant qu'on la lui montre: / «Ô mon Seigneur Jésus, ô Dieu de vérité, / Votre semblance était donc ainsi faite?»

Vers 1350 , les pèlerins chantaient un hymne composé à Avignon par le pape Jean XXII (1316-1334) :

22 Dom F. Cabrol, Abgar (La légende d') dans: Dictionnaire d'archéologie chrétienne et de liturgie, Paris: Letouzey et Ané, 1924, T. 1, col. 87-97. 
Je vous salue, ô saint visage de notre Rédempteur/ Où brille la figure de la divine splendeur. / Imprimé dans le tissu d'une blancheur de neige / Et donné à Véronique comme signe d'amour. / Je vous salue, visage du Seigneur, image bienheureuse. / Je vous salue notre gloire en cette rude vie.

L'image de la Véronique est le modèle absolu de toutes les icônes miraculeuses. Elle porte en elle des vertus divines et préfigure ainsi celle de la Vierge qui deviendra elle aussi rédemptrice. C'est l'une des fonctions essentielles des images pour le Moyen Age: assurer le salut. Ceci n'est pas à comprendre seulement sur un plan religieux mais aussi sur un plan psychologique. L'image est nécessaire à chacun pour se construire et pour évoluer. Comme l'a bien montré E. Cassirer, l'homme est un animal symbolicum. L'image est un principe central de toutes les activités humaines. Elle appartient au plus profond de notre vie mentale. $^{23}$

\section{La désacralisation littéraire de l’image}

Au Moyen Age, l'image est partout: c'est l'image peinte des miniatures ou des fresques murales, l'image sculptée dans la pierre des églises romanes, mais aussi l'image que portent les textes poétiques. Les troubadours ont su inventer un art de l'image dont le support est le mot (et la musique) mais qui suppose toujours une traduction visuelle directe. Avec la littérature profane, l'image se désacralise. Elle n'est plus figure du divin mais devient figuration du désir humain. L'un des Cantigas d'amigo du Martim Codax (Ondas do mar de Vigo) est parcouru d'une image verbale, sonore et visuelle: celle des vagues de la mer qui trahissent tout à la fois l'absence de l'amant, la nostalgie de sa présence et l'espoir des retrouvailles.

Les ondulations de la musique, le jeu des vers refrains qui imitent la houle, le jeu des rimes (igo-igo-do-do-do-do-igo-iro-edo-ado-ado-edo) font alterner sous le vocalisme du o commun à tous les vers des variations consonantiques symbolisant le flux et le reflux des vagues. Elles trahissent tout un jeu formel initié par l'image de la mer à la

\footnotetext{
${ }^{23}$ Cassirer, E. - La philosophie des formes symboliques, Paris: Minuit, 1972, 3 vol.
} 
fois changeante et monotone. Cette image de la mer est le support du fantasme amoureux de la dame. C'est autour de l'image subjective et intériorisée de la mer que se déploie le chant poétique.

On dit parfois que le Moyen Age a découvert l'amour. Il serait plus exact de dire qu'il a découvert la nature fantasmatique du phénomène amoureux. C'est ce que déclare Giorgio Agamben dans un brillant essai sur la parole et le fantasme dans la culture occidentale: «La découverte médiévale de l'amour, sur quoi l'on a si souvent discuté, parfois hors de propos, est en vérité la découverte de l'irréalité de l'amour: c'est-à-dire de son caractère fantasmatique. La connexion entre désir et fantasme, que l'antiquité avait à peine pressentie dans le Philèbe de Platon, le Moyen Age la pousse jusqu'à ses extrêmes conséquences: c'est à cela que tient la nouveauté de son éros, et non à une prétendue absence de spiritualité érotique du monde classique». ${ }^{24}$ Les romans de Tristan et Yseut présentent une «salle aux ymages» où Tristan vient souvent se réfugier ${ }^{25}$. Le neveu du roi Marc a été séparé d'Yseut par décision du roi. Il ne peut plus la rencontrer ni même la voir. Pourtant, il est follement épris d'elle. Il sombre alors dans une mélancolie profonde qui est une réaction à la perte de l'objet aimé. L'absence d'Yseut provoque le désespoir de Tristan. La perte de l'objet aimé se transforme en une mutilation pour celui qui l'éprouve. Pour la compenser, Tristan a sculpté une «image» d'Yseut et il l'a cachée dans un lieu secret. A défaut de pouvoir contempler Yseut en personne, il se satisfait de ce simulacre. Son fantasme peut alors se laisser aller devant la statue comme devant une personne réelle. Lorsque l'objet de jouissance est inaccessible, il a besoin d'être remplacé. Telle est la fonction fétichiste de l'image pour Tristan: l'entretenir dans l'illusion d'une présence réelle (celle d'Yseut) afin de mieux supporter le désir inassouvi qui le déprime. Il s'adresse à la statue comme une personne réelle. Il lui parle et lui confie sa pensée:

Et les deliz des granz amors

E les travaus et lor dolurs

E lor paignes et lor ahans

\footnotetext{
${ }^{24}$ Agamben, G. - Stanze. Parole et fantasme dans la culture occidentale, Paris: Bourgois, 1981, p. 137.

${ }^{25}$ Walter, Ph. - Le Gant de verre. Le mythe de Tristan et Yseut, La Gacilly: Artus, 1990.
} 
Recorde a l'himage Tristrans (Turin, v. 1-4) ${ }^{26}$

«Les plaisirs des grands amours, leurs tourments et leurs douleurs, leurs peines et leurs souffrances, Tristan les rappelle à l'image. »

Por iço fist ceste image

Que dire li voet son corage

Son bon penser et sa fole errur,

Sa paigne, sa joie d'amor (Turin, v. 45-48)

«C'est pour cela qu'il fit cette statue, parce qu'il voulait lui dire ses sentiments, ses bonnes pensées et son trouble insensé, sa peine, sa joie d'amour».

L'image est présentée comme l'œuvre artistique de Tristan. Elle est une invention esthétique soulignant la nature fantasmatique de toute création. Peinture, musique et poésie naissent d'un désir inassouvi. Elles trouvent dans l'image sonore ou visuelle le support de toute création poétique. En d'autres termes, l'image devient le support d'un imaginaire, c'est-à-dire d'une pensée différente de celle des concepts, une pensée que l'on pourra qualifier de symbolique et qui mobilise l'affectivité plus que la rationalité. L'image sacralise la vie intérieure de l'individu.

Dans le monde moderne, l'image a subi une forte revalorisation. Freud a donné une caution scientifique aux intuitions des poètes romantiques, fasciné par les rêves et l'irrationnel et persuadés que les images ne relèvent pas d'une agitation déraisonnée de l'esprit. Les images (des rêves) par exemple sont pour lui des messages de l'inconscient. Elles livrent des messages qu'il faut savoir décrypter si nous ne voulons pas sombrer dans la névrose ou la psychose. G. Bachelard va encore plus loin en affirmant que toute notre vie mentale est d'abord faite d'images qui évoluent soit en créations poétiques, soit en intuitions scientifiques. Dans tous les cas, pour le monde moderne, l'image

\footnotetext{
${ }^{26}$ Tristan et Yseut. Les poèmes français, édités et traduits par Ph. Walter, Paris: Le Livre de poche, 1989.
} 
possède une fonction révélatrice. Elle est riche de significations profondes. Elle n'est pas qu'un simulacre purement extérieur car elle est liée à notre subjectivité. On rappellera d'ailleurs que le mot imagination désigne, dès le XIIe siècle, les images du rêve. L'image est un produit humain et l'homme se projette en elle intentionnellement. Elle est une épiphanie de l'être.

\section{L’image comme mémoire}

Si l'image joue un tel rôle, c'est parce qu'elle touche à la fois au passé et à l'avenir de l'humanité. Par rapport au passé, elle est un mémorial. Elle n'est pas seulement rattachée à la vie du sujet qui l'a produite. Elle se rattache aussi à l'histoire d'une culture ; elle témoigne à ce titre de l'histoire de l'humanité. Par rapport à l'avenir, l'image est aussi une promesse de bonheur: elle est un facteur d'équilibre psychique et social. Elle libère l'individu des contraintes de l'espace et du temps car elle suppose un acte de libre contemplation. Elle devient ainsi promesse de rayonnement intérieur. Fondamentalement, elle nous arrache à l'angoisse de la mort. Comme l'a bien souligné Gilbert Durand, dans l'espèce humaine, l'imaginaire a un rôle anti-dépresseur; il a pour fonction d'exorciser la peur de la mort. ${ }^{27}$ L'image appelle une transcendance qui n'est pas nécessairement divine mais qui, comme l'art selon Malraux, construit un «antidestin». L'art est une victoire sur la mort. Il nous arrache au non-sens de l'existence et à ses tragédies.

La plus célèbre des images de Chrétien de Troyes est celle immortalisée dans les trois gouttes de sang sur la neige de son Conte du Graal. Ces gouttes de sang illustrent le passage de l'image à l'imaginaire. Après avoir vu le graal, Perceval se trouve sur une prairie enneigée. Soudain autour de lui, un faucon attaque une oie et la blesse. Trois gouttes de sang s'échappent des flancs de l'oiseau et tombent sur la neige. L'association du vermeil et du blanc compose alors une douce «semblance» dans la contemplation de laquelle Perceval s'abîme. Il muse sur cette image pendant toute une matinée. Rien ne peut le distraire de ce penser et de cette jouissance muette.

\footnotetext{
${ }^{27}$ Durand, G. - L'imaginaire. Essai sur les sciences et la philosophie de l'image, Paris: Hatier, 1994.
} 
Cette image, maintes fois étudiée, est un bel exemple du basculement de l'image dans l'imaginaire. A travers l'imaginaire en effet, ce qui s'exprime c'est le pouvoir des images sur notre esprit, leur capacité à cheminer en nous et à creuser du sens au-delà des apparences. Cette image a une histoire et une mémoire. On la retrouve dans plusieurs contextes qui n'ont aucun lien direct avec le roman de Chrétien de Troyes comme si elle refaisait surface à des moments différents de la culture occidentale. Par exemple dans le conte de Blanche-neige:

«En plein hiver, quand les flocons descendaient du ciel comme des plumes et du duvet, une reine qui était assise et cousait devant une fenêtre qui avait un encadrement en bois d'ébène, noir et profond. Et tandis qu'elle cousait négligemment tout en regardant la belle neige au-dehors, la reine se piqua le doigt avec son aiguille et trois petites gouttes de sang tombèrent sur la neige. C'était si beau, ce rouge sur la neige, qu'en le voyant, la reine songea: "Oh! si je pouvais avoir un enfant aussi blanc que la neige, aussi vermeil que le sang et aussi noir de cheveux que l'ébène de cette fenêtre!" Bientôt après, elle eut une petite fille qui était blanche comme la neige, vermeille comme le sang et noire de cheveux comme le bois d'ébène, et Blanche-Neige fut son nom à cause de cela. Mais la reine mourut en la mettant au monde.»

L'image (le motif) est ici un appel. Il fait signe et il fait sens. Il s'inscrit dans une longue histoire qui parcourt les civilisations. Dans une étude peu connue, Emmanuel $\operatorname{Cosquin}^{28}$ a suivi ces variations du motif du sang sur la neige dans les contes de différents pays et à toutes les époques sans omettre l'épisode du Conte du Graal. Il en retrouve la trace dans le Pentamerone de Basile (un roi parti à la chasse découvre un corbeau mort sur une dalle de marbre. En voyant le sang sur la blancheur du marbre, il souhaite une épouse au visage aussi blanc et rouge que la dalle et aux cheveux aussi noirs que le corbeau). Le Livre de Leinster en Irlande présente une histoire comparable (Deirdre voit un corbeau qui boit du sang mélangé à de la neige. Elle dit à une magicienne qui se trouve là qu'elle souhaite un mari aux cheveux noirs comme le corbeau, aux joues rouges comme le sang et au corps blanc comme la neige et son vœu

\footnotetext{
${ }^{28}$ Cosquin, E. - Les contes indiens et l'Occident, Paris: Champion, 1922, p. 218-245.
} 
s'accomplit). On pourrait allonger la liste indéfiniment. En réalité, ce qui s'exprime à travers cette scène colorée, c'est le désir d'un (ou d'une) partenaire (ou enfant) surnaturel (féerique).

La structure interne de l'image (ainsi que certains éléments de sa signification) n'apparaissent qu'au terme d'une comparaison de contextes en dissociant le moins possible le faisceau des motifs de base. Un chasseur aperçoit un animal qui laisse des traces de sang sur la neige après une agression. Trois couleurs sont présentes: le rouge (sang), le blanc (neige) et parfois le noir. La conjonction de ces couleurs lui donne l'occasion de formuler un vœu qui semble prendre le plus souvent une valeur performative: le fait de l'énoncer le provoque. Or, cette propriété de la parole qu'on pourrait dire magique est caractéristique des êtres féeriques. Elle permet de penser que l'être ainsi appelé à devenir le partenaire du héros ou de l'héroïne (car le récit peut s'inverser au féminin) est lui aussi d'essence féerique. Le motif des gouttes de sans sur la neige montre à l'évidence comment la littérature médiévale nous ramène toujours sur le chemin du mythe, à des images au passé archaïque à partir desquelles l'imaginaire littéraire peut se déployer à l'infini. Il existe bien une fonction mythopoétique des images, comme l'a bien souligné Jean-Jacques Wunenburger. C'est cette fonction qui conduit à reconnaître la légitimité de la notion d'imaginaire. ${ }^{29}$

Lorsqu'on parle d'image, il faut prêter attention au support de celle-ci. L'image verbale (créée par les mots) n'est pas l'image visuelle (reçue par nos sens) qui elle-même n'est pas l'image picturale (réalisée par un peintre) qui elle-même n'est pas l'image mentale que nous nous représentons à partir d'une évocation plus ou moins consciente. Comme le rappelle Daniel Poirion ${ }^{30}$ : «L'évocation par le langage est indirecte. Dans un texte, nous n'avons pas affaire directement au monde «imaginaire» mais seulement à la fabrication des images, par un vocabulaire, des procédés de grammaire et de rhétorique, des formules. Il n'y a d'image à proprement parler que dans la pensée du lecteur ou de l'auditeur. Image au second degré, provoquée par une certaine technique de l'écriture (descriptive par exemple ou allégorique). Il faudrait donc se rappeler ce décalage

\footnotetext{
${ }^{29}$ Wunenburger, J. J. - La vie des images, Presses Universitaires de Grenoble, 2002.

${ }^{30}$ Poirion, D. - «Du sang sur la neige: nature et fonction de l'image dans le Conte du Graal », dans: R. Cormier éd., Voices of conscience, Philadelphie: Temple University Press, 1977, p. 143-164.
} 
essentiel quand on cherche à expliquer l'imagination poétique ou romanesque en fonction des structures et des significations d'un système dit «symbolique». C'est souligner la part décisive que joue l'herméneutique (autrement dit le lecteur) dans cette construction du sens. Le sens n'est pas déjà là: il est toujours construit et reconstruit par le lecteur.

\section{Conclusion: Lector in fabula et imagine}

L'image des trois gouttes de sang sur la neige offre un bel exemple de sémiologie complexe à l'œuvre dans un texte. C'est probablement le meilleur exemple pour tenter de définir le passage de l'image à «l'imaginaire» médiéval, c'est-à-dire un processus de symbolisation à partir d'une image visuelle. Un grand principe préside à cette émergence du symbole: l'activation de l'imaginaire présuppose une illusion perceptive. L'imaginaire résultera toujours d'une surinterprétation d'une perception. Lorsque Perceval aperçoit trois gouttes de sang sur la neige, il en reste à une perception mais lorsque le narrateur déclare que Perceval «s'imaginait dans son ravissement voir les fraîches couleurs du visage de sa belle amie» (Conte du Graal, éd. D. Poirion, Pléiade, p. 789), l'imaginaire entre en jeu car une interprétation de la perception a lieu. Une signification se surimprime sur l'image. Perceval entraîne alors le lecteur dans un jeu herméneutique car le héros est le substitut du lecteur dans la fiction. Lector in fabula dirait Umberto Eco. C'est ce jeu du personnage-lecteur qui a inauguré la longue chaîne des interprétations du roman et fondé le mythe du graal. Tel est le jeu de bascule fondamental entre perception et interprétation. Toute image est en attente d'interprétation et l'interprétation change selon les personnes et les époques. Sous son apparente objectivité, l'image est en réalité un miroir de notre subjectivité. La littérature peut se définir comme l'exploration illimitée du pouvoir des images. Le langage des images dit toujours autre chose que le discours rationnel. Il y a toujours un écart irréductible entre le thème imagé et la thèse idéologique qui ne doit pas être artificiellement réduit par notre esprit de système. Car, comme le dit Daniel Poirion «c'est pour le vivre (cet écart) au besoin dans la contradiction, qu'on a écrit et qu'on lit encore ces histoires qui nous rendent «pensifs» et entretiennent notre rêverie». 


\section{COMO CITAR ESTE ARTIGO}

\section{Referência electrónica:}

WALTER Philippe - “De l'image à l'imaginaire médiéval”. Medievalista [Em linha]. No13, (Janeiro - Junho 2013). [Consultado dd.mm.aaaa]. Disponível em http://www2.fcsh.unl.pt/iem/medievalista/MEDIEVALISTA13/walter1303.html.

ISSN 1646-740X.

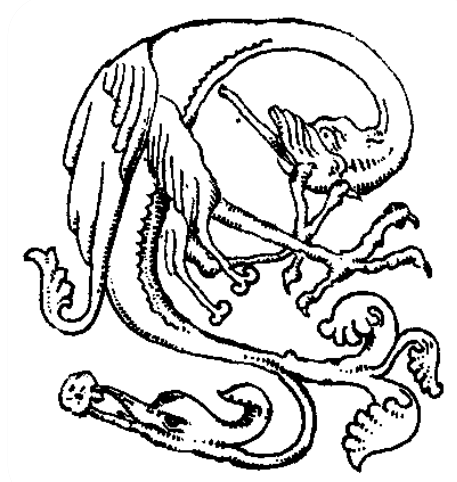

